\title{
The acute influence of stretching in muscle resistance strength: transversal intervention study.
}

\author{
Jefferson Petto ${ }^{1,4,6,7}$, Tarcísio de Almeida Cruz², Diego Silva Patrício ${ }^{3,6,8}$, Marvyn de Santana do Sacramento ${ }^{4}$, \\ André Ricardo da Luz Almeida ${ }^{5}$, Luís Alberto Bastos de Almeida², Alan Carlos Nery dos Santos ${ }^{1,7}$, \\ Diego Passos Diogo 1,2,6,
}

\begin{abstract}
Background: Muscle stretching is often applied as a preparation for resistance training, however, the literature has diverged as to its response on muscular endurance strength. Objective: to show whether the intensity and time of muscle stretching modifies the resistance strength. Methods: The study included 30 healthy young males aged $22 \pm 2.5$ years, who had been practicing neuromuscular exercise for at least six months. Participants took a maximum load prediction test (MLPT) on the bench press and after one week returned to the draw and execution of the: Stretch Protocol (SP) - 60 seconds at maximum amplitude without assistance; Flexing Protocol (FP) - 60 seconds of stretching with external assistance; and Base Protocol (BP) - immediate exercise. All participants went through all protocols always performed on Mondays, performing the maximum repetitions with $70 \%$ of the load obtained in the MLPT. To compare the results of the different days of collection was used the ANOVA with TUKEY post-hoc. All analyzes adopted a value of p $\leq 0.05$ as significant. Results: BP showed significantly higher results than in SP and FP. There was no statistical difference between SP and FP. Conclusion: 60 seconds of passive muscle stretching causes a decrease in muscle resistance strength, regardless of its intensity.

Keywords: Flexibility; Stretch; Resistance Training; Physical Exercise; Neuromuscular Exercise.
\end{abstract}

\section{INTRODUCTION}

The attribution of resistance training (RT), also called neuromuscular, for health maintenance is increasing and its practice is almost always present, being used in sports training programs as well as functional rehabilitation ${ }^{(1)}$. Given their importance, it is paramount to deeply understand their prescription variables, volume variables (number of sets and repetitions, weekly frequency and number of exercises per muscle group) and intensity variables (load, speed of execution and interval between sets). However, there are other variables that are extrinsic to the prescription of RT that directly interfere with the response caused by it, such as muscle stretching. Muscle stretching is a technique that commonly precedes RT and is the point of much discussion between professionals and practitioners. Among the discussions that revolve around this variable, one has caught the researchers' attention - the interference in the performance of strength, power and endurance during the execution of RT, when preceded by muscle stretching ${ }^{(2,3)}$. There are many ways to classify and apply muscle stretching. According to Dantas ${ }^{4}$ muscle stretching can be classified as lengthening and flexing. Lengthening is defined as a technique aimed at maintaining range of motion as it is performed within the natural range of the joint. Whereas flexion is a technique that aims to gain joint amplitude and is performed exceeding the natural joint limit of the subject, with external help seeking to reach the largest possible $\operatorname{arch}^{(4,5)}$. We therefore consider flexing as a more intense form of muscle stretching.

A review carried out by Peck et al. ${ }^{(6)}$ demonstrated that the static stretching preceding the various sports may result in decreased muscle strength; however, this review did not stipulate the dichotomy adopted by Dantas ${ }^{(4)}$ between lengthening and flexing. In a study by our research group, it was evaluated the acute effect of lengthening and flexion on the resistance strength of young people who had previous experience in RT. We sought to investigate whether applying muscle stretching could cause a different response. It was observed that both lengthening and flexing did not reduce resistance strength when previously applied to a series of bench press ${ }^{(7)}$. However, we used a muscle stretching time of less than 40 seconds. According to Kisner ${ }^{1}$, the time required to reach the plastic phase of the muscle cell during a stretching job is 40 seconds. Below this time the cell only reaches the elastic phase, in which the muscle cell returns to its initial state after exposure to stretching, while in the plastic

\footnotetext{
Corresponding author: Marvyn de Santana do Sacramento. R. Rio Grande do Sul, 356 - Pituba, Salvador - BA, 40830-140. marvynsantana@gmail.com. 71 98657-2849.

${ }^{4}$ Departamento de Fisioterapia- Centro Universitário Social da Bahia (UNISBA), Salvador, BA, Brasil.

Full list of author information is available at the end of the article.
}

Financial support: nothing to declare.

Submission date 05 March 2019; Acceptance date 18 April 2019; Publication date 21 December 2019 
phase (over 40 seconds) there is a change in the structural conformation of the cell, which takes longer to return to its basal state. Therefore, in the present study we decided to test the hypothesis that the previous application of lengthening and flexion in RT can decrease the resistance strength when the plastic phase of the muscle cell is reached.

\section{METHODS}

\section{Lineation}

The research was characterized as a controlled intervention study. It was selected 30 students from the Departamento de Saúde da Universidade Estadual de Feira de Santana. It was included healthy young males aged 18 to 28 years with practical experience of at least six months of RT. and it was excluded subjects who had any osteomioarticular limitation, pain or inflammation or any disease that affected their physical performance. Weekly practice volume and practice time were not used as sample eligibility criteria. To calculate the sufficiency of the sample, a pilot study with 3 subjects was carried out, in which it was found a minimum difference between the averages of the proposed protocols of three and a standard deviation of the error of 2.2. Considering the normal data distribution, for a power of $80 \%$ and alpha of $5 \%$, it was reached the necessary sample of 23 individuals.

\section{Ethical aspects}

Throughout the study, the guidelines on human research in the Helsinki Declaration and National Health Council Resolution $466 / 12$ were observed. This study was submitted and approved by the Research Ethics Committee with CAAE: 0054.0690.077-06. All subjects received detailed information about the risks and benefits involved in the procedures and signed an informed consent form. The purpose of the research was not revealed to the volunteers so that they did not suffer psychological influence in the execution of the tests.

\section{Intervention protocol}

All subjects were submitted to three exercise protocols: the Lengthening Protocol (LP), which performed a stretching session before exercise, the Flexing Protocol (FP), in which the subjects were submitted to a stretching session before the exercise, and the Basic Protocol (BP), in which the subjects were not submitted to any muscle stretching technique before the resistance exercise performed in the bench press. Maximum load test and protocols were performed at the sports academy "Fisicu`s Academia" in Feira de Santana, BA, Brazil.

Firstly, the participants underwent the maximum load prediction test described by Lombardi( ${ }^{(8)}$ and Adams ${ }^{(9)}$. In this test, the body mass of each individual is multiplied by the coefficient established for the bench press exercise, so that the value found was adopted as the test load (TL). Each participant performed as many repetitions as possible with the determined TL for the movement, and finally, the load correction was performed based on the training load adjustment table, developed by Lombardi( ${ }^{(8)}$. For the exercise, $70 \%$ of a Maximum Repeat (MR) was used.

Seven days after the maximum load test, participants returned to the gym to perform the proposed protocols. A simple draw was performed to determine which of the protocols (LP, FP or BP) the volunteer would perform on the day.

The LP was based on the concept of stretching described by Dantas $^{(4)}$, in which the technique is actively performed. Thus, the evaluator asked each volunteer to position themselves prone on the mat, with the lower limbs extended and the head in neutral position. After positioning, the subjects were asked to perform bilateral maximal horizontal abduction with the glenohumeral joint in external rotation and the elbow in complete extension. The trunk, lower limbs and head remained in full contact with the surface. This position was held static for 60 seconds. It was decided to keep the stretching stimulus for 60 seconds to ensure that the plastic phase of muscle deformation was reached, which according to Kisner and Colby ${ }^{(1)}$ is reached after 40 seconds.

After stretching the pectoralis major and deltoid anterior portion, the volunteer performed the stretching of the triceps brachii. The subject was oriented to stand in sedestation on the bench press, with the feet in full contact with the ground, erect spine and look fixed on the horizon. Maximum shoulder and elbow flexion with one of the upper limbs was requested, maintaining the position in a static way for a period of 60 seconds, repeating the technique on the contralateral limb. The deltoid (anterior portion), pectoralis major and triceps brachii muscles were the muscles chosen for stretching because they are the main agonists of the supine movement ${ }^{(10)}$. The position used to perform the stretching can be seen in figure 1 . Then, the volunteer was submitted to the supine straight exercise.

The FP was performed in the same way as the LP, being added the application of external force by the evaluator. The force was applied passively, static and holding position for a period of 60 seconds, bringing the joint to a comfortable level of stretching. Figure 2 illustrates the flexion applied to the FP. As on the day of $L P$, immediately after flexion, the bench press exercise was performed. In $\mathrm{BP}$, the volunteers performed the bench press exercise without the previous execution of any type of warm-up and/or muscle stretching techniques.

It was decided to keep seven days of difference between the protocols so that they were always performed on the same day of the week (Monday). Subjects were instructed to follow their exercise routine during the research period, performing all exercises they were used to performing during their weekly training program, and were asked not to engage in any additional physical activity on weekends during the collection period. Importantly, the protocols performed by the volunteers 

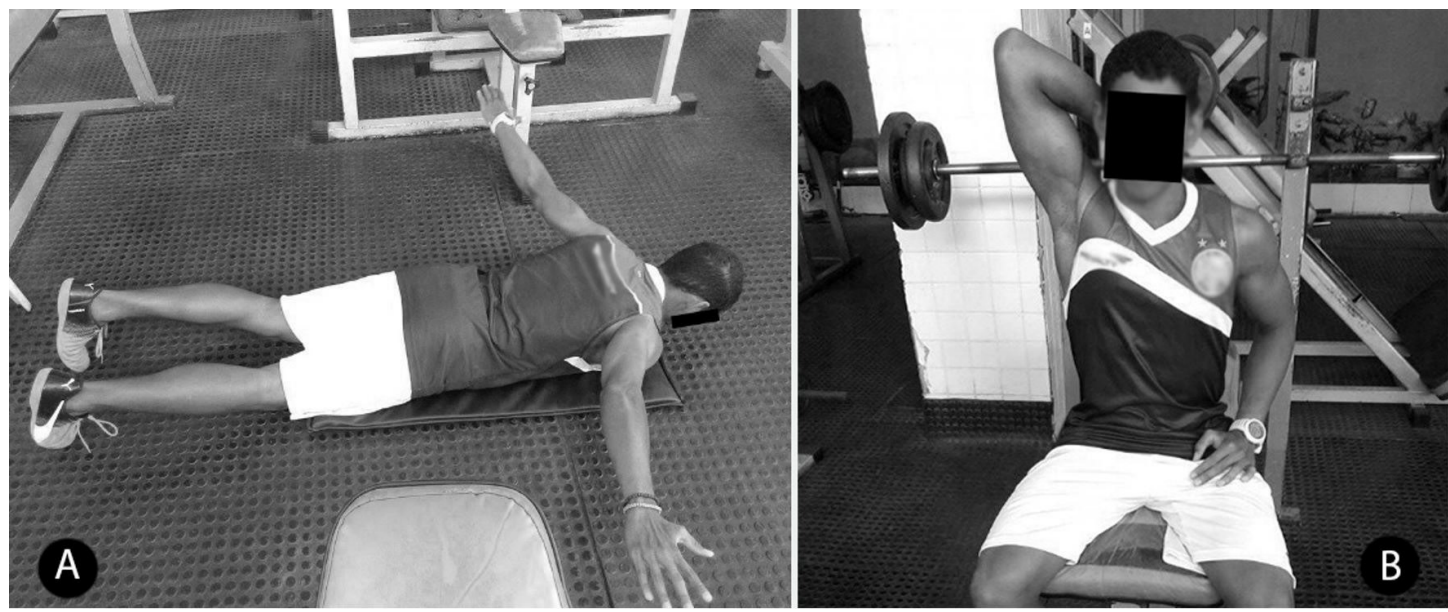

Figure 1. Stretch protocol. Note: A) Chest Stretch Protocol. B) Triceps Stretch Protocol.
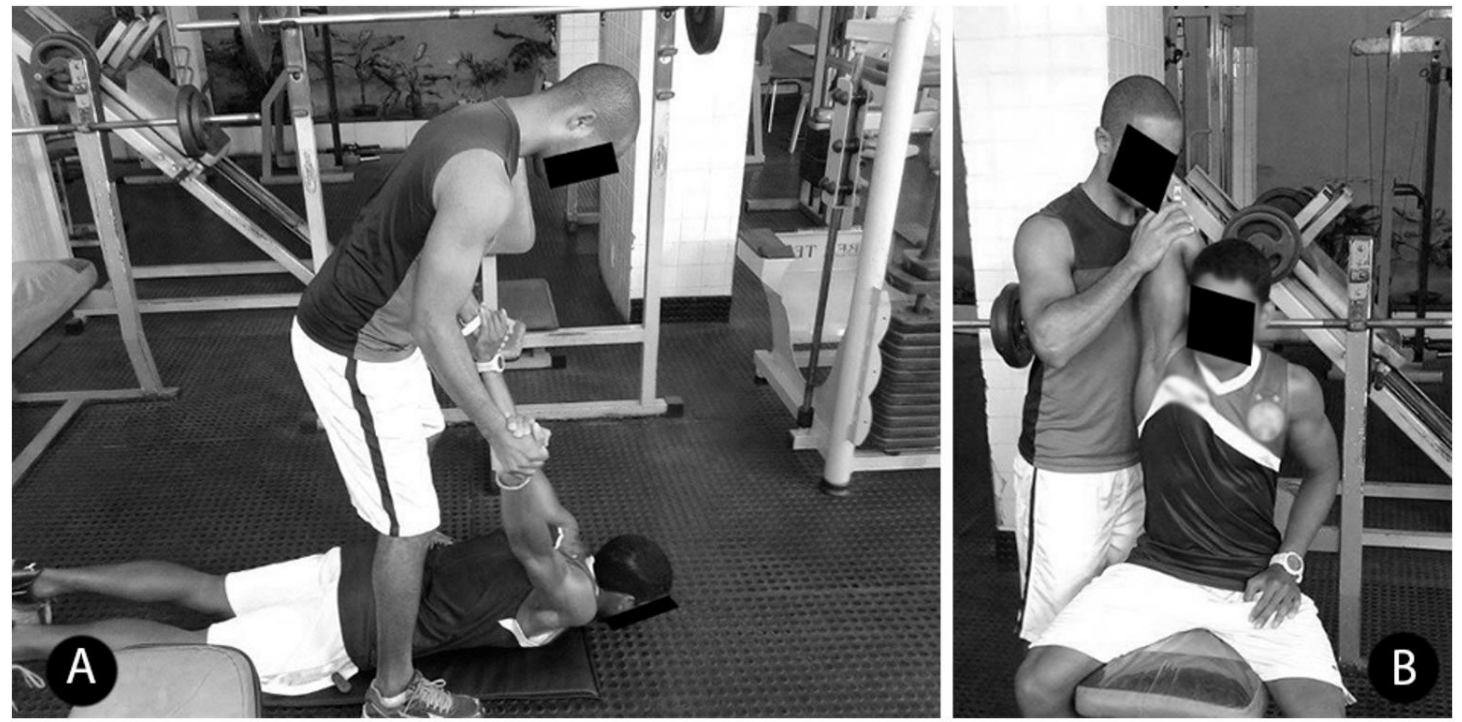

Figure 2. Flexion protocol. Note: A) Pectoral Flexion Protocol. B) Triceps Flexion Protocol.

obeyed the simple randomization, which was performed on the day the participants performed the protocols.

Regarding the technique of performing the bench press exercise, the protocol was performed as follows: each volunteer was positioned supine on the bench press with the knee and hip flexion joints, supporting the sole of the feet on the bench. Each participant was helped by an assistant to remove the bar from the stand when starting the exercise. Movement was performed over the full range of motion at an approximate cadence of 2:3 seconds for each phase of the movement (concentric and eccentric respectively). The control of the execution speed of the movement was done verbally. The evaluator controlled the runtime by a digital timer (SportWatch XL021). The onset of fatigue was considered when compensatory movements were observed in the execution of the exercise and/or when the concentric phase failed. For safety reasons three assistants were positioned around the bar: two at the ends and one positioned behind the bench. The research subjects were not verbally and/or visually stimulated during the period preceding the exercise or during the performance.

To evaluate the resistance strength of the participants were recorded how many repetitions they performed on each collection day. The number of repetitions reached each day served as a parameter to analyze the effect of lengthening and flexion on the muscular endurance strength.

\section{Statistical Analysis}

Initially, to verify the distribution of data, symmetry and kurtosis tests and the Shapiro-Wilk test were applied. The values of the study variable presented normal behavior, therefore, they were described as mean and standard 
Table 1 - General characteristics of the sample $(n=30)$.

\begin{tabular}{lccc}
\hline & Minimum & Maximum & Mean \pm SD \\
\hline Age (years) & 18 & 28 & $22 \pm 2.5$ \\
Mass (kg) & 60 & 93 & $74 \pm 8.8$ \\
Height (cm) & 165 & 195 & $178 \pm 8.3$ \\
BMI (kg/m $)$ & 19 & 26 & $23 \pm 2.1$ \\
PMLT (kg) & 40 & 82 & $53 \pm 10.4$ \\
\hline
\end{tabular}

Note: SD - Standard Deviation; BMI - Body Mass Index; PMLT - Predicted Maximum Load Test.

Table 2 - Stretching and Basal Protocols

\begin{tabular}{cccc}
\hline & Basal & Lengthening & Flexing \\
\hline Mean \pm SD & $17 \pm 2.5^{*}$ & $15 \pm 3.1$ & $15 \pm 2.5$ \\
\hline
\end{tabular}

Note: The analysis of variance used a Tukey post-hoc criterion to compare the means of the three days of collection. Statistical significance was verified between basal and lengthening protocol and between basal and flexing protocol ( $P \leq 0.05)$. No significance was found between stretching and flexing protocols.

deviation. Once the normality was confirmed, for comparison between the results of the different days of collection, the ANOVA with post-hoc TUKEY was used. All the analyzes were performed in the BioEstat 5.0 statistical package (AnalystSoft Inc., Walnut, CA, USA), adopting a significance level of $5 \%$.

\section{RESULTS}

A total of 30 subjects were evaluated. Table 1 shows the means and standard deviation of the general characteristics of the sample and the value of the maximum predicted test load.

Table 2 shows the mean and standard deviation of the number of repetitions in the bench press, achieved in the three days of collection. We can observe statistically significant difference between the basal protocol compared to the lengthening and flexing protocols.

\section{DISCUSSION}

As mentioned in the introduction, our group in a previous study ${ }^{(7)}$ with similar protocol found that a stretching time of 30 seconds did not decrease the number of repetitions achieved by volunteers between protocols. It was initially demonstrated that the intensity of the stretch alone does not influence the strength of resistance. However, when performing the same protocol with a longer stretching time (60 seconds), in which we reached the plastic response of the muscle, we found that there was a difference in resistance strength, both on the day of lengthening and on the day of flexion. Therefore, it seems quite clear to us that what determines the fall in resistance strength is the time in which the muscle is exposed to stretching and not the intensity to which it is subjected. According to Kubo et al. ${ }^{(11)}$, the decrease in the resistance strength caused by the stretching work is mainly due to the alteration of the viscoelastic properties of the muscle-tendon unit caused by the flexibility techniques. This viscoelastic change reduces the passive tension and stiffness of the muscle, causing the ability to generate resistance, absolute and potency. This idea is shared by other researchers ${ }^{(12)}$. This fits in with the idea that a minimum stretch exposure time is required for the muscle cell and adjacent connective tissue to reach the plastic phase. In line with our study both Silveira et al. ${ }^{(13)}$ and Cardozo et al. ${ }^{(14)}$ observed no impact of muscle stretching work on resistance strength when different protocols were applied below 40 seconds.

Thus, regardless of which muscle tissue is being targeted primarily, conjunction with flexion or muscular with lengthening ${ }^{4}$, what impacts the performance of the resistance strength is the exposure time. This allows us to think that different muscle stretching techniques can be used prior to resistance workouts without diminishing performance, provided that a maximum exposure time of less than 40 seconds on each muscle is respected. However, consideration of the results needs to be done.

Firstly, it is important to point out that this idea it is time and not the intensity of stretching that decreased the resistance strength, does not apply to the absolute strength, because the absolute strength was not evaluated in this study. However, other studies indicate that the time of exposure to muscle stretching also influences the absolute strength. Ye et al. ${ }^{(15)}$ found a decrease in static absolute strength for the biceps brachii after a 100 second static muscle stretch. Arruda et al..$^{(16)}$, submitted 11 young men to passive stretching statically for 40 seconds and found strength reduction in the 10RM test when compared to the group that only performed warm-up. Tricoli and Paulo ${ }^{(17)}$, investigated the effects of a single session of static stretching exercises performed shortly before maximal strength performance. The subjects underwent 18 minutes of maximal passive stretching exercises in the legpress exercise, in which the maximum strength reduction was identified from the maximum repetition test. We also point out that the resistance strength test was applied immediately after muscle stretching. We did not know if the resistance strength would decrease if we waited a few minutes after the lengthening and flexing protocols to perform the bench press exercise. This is because other studies have shown that the interval between the application of muscle stretching and the evaluation of strength influences the result ${ }^{(18,19)}$.

Another point to be raised is that we evaluated subjects who had been practicing neuromuscular exercises for at least six months. We do not know if the result found in our study would be the same, with a population with no experience in resistance exercise. In addition, we evaluated the acute effect of just one muscle stretching session (lengthening or flexing) on resistance strength. We do not know whether with chronic application of lengthening or flexing, we would get a different result. Possibly the result would be the same. This is because, in the study conducted by Junior et al. (19), the authors identified that muscle stretching (50 seconds) 
in a population of sedentary individuals with no experience with neuromuscular exercises caused a decrease in resistance strength and total exercise volume as an acute effect. Also in this study, the authors found that there was a negative impact on muscle hypertrophy as a chronic effect (10 weeks).

Despite the methodological limitations, some of which are mentioned above, it can be highlight that this study corroborates the advancement of professional practice and science, since we did not find studies that had evaluated what we investigated in this study and in the previous study $y^{(7)}$ - the difference in the effect of the form of application of muscle stretching (lengthening and flexing). Moreover, the result found here allows us to better understand the divergences found in the results of previous studies by other researchers.

For example, Nelson et al. ${ }^{(21)}$ observed a decrease in the number of repetitions when they subjected volunteers to passive stretching before neuromuscular exercise. Whereas Franco et al. ${ }^{(22)}$ did not identify any difference in resistance strength performance when applying static stretching. Based on the results of our study, we can explain the difference found in these studies by the time of application of muscle stretching, since Nelson et al. ${ }^{(21)}$ used 15 minutes and Franco et al. ${ }^{22}$ used 20 seconds on each muscle.

Finally, it is essential to point out that our findings and those of the works cited here refer to techniques that do not use Proprioceptive Neuromuscular Facilitation (PNF). That is because studies show controversial results when using PNF, in which shorter exposure times caused substantial decreases in resistance strength and strength ${ }^{(15)}$. In short, we believe that any flexibility technique (including PNF) that reaches the plastic phase of the muscle, applied immediately before RT, results in decreased resistance force impacting the subject's acute performance. Possibly, other works will still be produced, as there are still many questions to be answered regarding this theme and then the knowledge will be expanded. Therefore, it is necessary that professionals who use flexibility techniques, be aware of new knowledge and evaluate in the light of scientific evidence the best ways to use these techniques, reflect on their effects (positive and negative) and from that reflection know which patients or clients to apply them and the best time to apply them.

\section{CONCLUSION}

Based on the results obtained in this study, we conclude that 60 seconds of passive muscle stretching causes a decrease in muscular resistance strength. Therefore, it is hypothesized that the effect of stretching on the resistance strength is independent of its intensity (lengthening or flexing).

\section{AUTHORS' CONTRIBUTIONS:}

JP and TAC elaborated the study design; MSS, ARLA, LABA and ACNS performed data collection; DSP and JP performed the statistical analysis; ACNS, DPD and JP conducted the critical intellectual review of the manuscript. All authors read and approved the final manuscript.

\section{CONFLICT OF INTEREST:}

nothing to declare.

\section{AUTHORS DETAILS}

${ }^{1}$ Departamento de Fisioterapia- Escola Bahiana de Medicina e Saúde Pública (EBMSP), Salvador, BA, Brasil. ${ }^{2}$ Departamento de Educação Física- Universidade Estadual de Feira de Santana (UEFS). Feira de Santana, BA, Brasil. ${ }^{3}$ Departamento de Fisioterapia- Centro Universitário CESMAC (FEJAL), Maceió, Alagoas, Brasil. ${ }^{5}$ Departamento de Educação Física- Faculdade Nobre (FAN), Feira de Santana, BA, Brasil. ${ }^{6}$ Departamento de Fisioterapia. Faculdade Adventista da Bahia (FADBA), Cachoeira, BA, Brasil. ${ }^{7}$ Grupo de Pesquisa Ciências da Saúde em Fisioterapia. Universidade Salvador (UNIFACS), Feira de Santana, BA, Brasil. ${ }^{8}$ Departamento de Fisioterapia- Universidade Estadual da Paraíba (UEPB), Campina Grande, Paraíba Brasil.

\section{REFERENCES}

1. Kisner C, Colby LA. Exercícios terapêuticos: fundamentos e técnicas. 4. ed. Barueri: Manole, 2005.

2. Alizadeh EL, Çetin E. Duration Dependent Effect of Static Stretching on Quadriceps and Hamstring Muscle Force. Sports (Basel). 2018;6(1): 24.

3. Lima CD, Brown LE, Wong MA, Leyva WD, Pinto RS, Cadore EL. Acute effects of static vs. Ballistic stretching on strength and muscular fatigue between ballet dancers and resistance-trained women. J. Strength Cond. Res. 2016; 30(11): 3220-7.

4. Dantas EHM. Alongamento e flexionamento. 5. ed. Rio de Janeiro: Shape, 2005.

5. Iwata M, Yamamoto A, Matsuo S, Hatano G, Miyazaki M, Fukaya T et al. Dynamic Stretching Has Sustained Effects on Range of Motion and Passive Stiffness of the Hamstring Muscles. J Sports Sci Med. 2019; 11; 18(1): 13-20.

6. Peck E, Chomko G, Gaz DV, Farrell AM. The Effects of Stretching on Performance. Sports Med. Rep. 2014; 13(3): 179-85.

7. Diogo DP, Novais LS, Gomes RL, Almeida Neto AB, Santos ACN, Petto J. Diferentes intensidades do estiramento muscular sobre a resistência de força de jovens ativos. R. Bras. Ci. e Mov. 2014; 22(4): 156-61.

8. Lombardi VP. Beginning weight training: the safe and effective way. Dubuque: W. C. Brown, 1989.

9. Adams GM. Exercise physiology laboratory manual. Boston: McGraw Hill, 1998.

10. Escamilla RF, Lewis C, Pecson A, Imamura R, Andrews JR. Muscle Activation Among Supine, Prone, and Side Position Exercises With and Without a Swiss Ball. Sports Health. 2016; 8(4): 372-9.

11. Kubo K, Kanehisa H, Kawakami Y, Fukunaga T. Influence of static stretching on viscoelastic properties of human tendon structures in vivo. J Appl Physiol. 2001;90:520-7.

12. Serpa E, Vilela Júnior GB, Marchetti PH. Aspectos biomecânicos da unidade músculo tendínea sob efeito do alongamento. Revista CPAQV. 2014; 6(1): $1-14$.

13. Silveira RN, Farias JM, Alvarez BR, Bif R, Vieira J. Efeito agudo do alongamento estático em músculo agonista nos níveis de ativação e no desempenho da forca de homens treinados. R Bras Med Esporte. 2011; 17(1): $26-30$

14. Cardozo G, Torres JB, Dantas EHM, Simão R. Comportamento da força muscular após o alongamento estático. Revista Treinamento Desportivo. 2006; 7(1): 73-6.

15. Ye X, Beck TW, Wages NP. Influence of prolonged static stretching on motor unit firing properties. Nerve \& Muscle. 2016; 53(5): 808-17.

16. Arruda FLB, Faria LB, Silva V, Senna GW, Simão R, Novaeis JS et al. A influência do alongamento no rendimento do treinamento de força. Treinamento Desportivo. 2006; 7(1): 1-5 
17. Tricoli V, Paulo AC. Efeito agudo dos exercícios de alongamento sobre o desempenho da força máxima. R. Bras. Atividade Física e Saúde. 2002; 7(1): 6-13.

18. Paz GA, Leite $T$, Maia MF, Lima AF, Coelho PP, Simão R et al. Influência do intervalo de recuperação entre alongamento e treinamento de força. ConScientiae Saúde. 2013; 12(3): 362-70.

19. Kendall BJ. The Acute Effects of Static Stretching Compared to Dynamic Stretching with and without an Active Warm up on Anaerobic Performance. Int J Exerc Sci. 2017;1;10(1):53-61.
20. Junior RM, Berton R, Souza TMF, Chacon-Mikahil MPT, Cavaglieri CR. Effect of the flexibility training performed immediately before resistance training on muscle hypertrophy, maximum strength and flexibility. Eur J Appl Physiol. 2017;117:767.

21. Nelson AG, Kokkonen J, Arnall DA. Acute muscle stretching inhibits muscle strength endurance performance. J Strength Cond Res. 2005;19(2):338-43.

22. Franco BL, Signorelli GR, Trajano GS, de Oliveira CG. Acute effects of different stretching exercises on muscular endurance. J Strength Cond Res. 2008;22(6):1832-7. 